\title{
Improved Garbled Circuit: Free XOR Gates and Applications
}

\author{
Vladimir Kolesnikov ${ }^{1}$ and Thomas Schneider ${ }^{2, \star}$ \\ ${ }^{1}$ Bell Laboratories, 600 Mountain Ave. Murray Hill, NJ 07974, USA \\ kolesnikov@research.bell-labs.com \\ ${ }^{2}$ Horst Görtz Institute for IT-Security, Ruhr-University Bochum, Germany \\ thomas.schneider@trust.rub.de
}

\begin{abstract}
We present a new garbled circuit construction for two-party secure function evaluation (SFE). In our one-round protocol, XOR gates are evaluated "for free", which results in the corresponding improvement over the best garbled circuit implementations (e.g. Fairplay 19]).

We build permutation networks [26] and Universal Circuits (UC) 25] almost exclusively of XOR gates; this results in a factor of up to $4 \mathrm{im}$ provement (in both computation and communication) of their SFE. We also improve integer addition and equality testing by factor of up to 2 .

We rely on the Random Oracle (RO) assumption. Our constructions are proven secure in the semi-honest model.
\end{abstract}

\section{Introduction}

Two-party general secure function evaluation (SFE) allows two parties to evaluate any function on their respective inputs $x$ and $y$, while maintaining privacy of both $x$ and $y$. SFE is (justifiably) a subject of immense amount of research, e.g. 272817. Efficient SFE algorithms enable a variety of electronic transactions, previously impossible due to mutual mistrust of participants. Examples include auctions 21684, contract signing [7, distributed database mining 12 16, etc. As computation and communication resources have increased, SFE has become truly practical for common use. Fairplay [19] is a full-fledged implementation of generic two-party SFE with malicious players. It clearly demonstrates feasibility and efficiency of SFE of many useful functions, represented as circuits of up to $\approx 10^{6}$ gates. Today, generic SFE is a relatively mature technology, and even improvements by a small factor are non-trivial and are most welcome.

One area of SFE that especially benefits from our work is the SFE of private functions (PF-SFE). It is an extension of SFE where the evaluated function is known only by one party and needs to be kept secret (i.e. everything besides the size, the number of inputs and the number of outputs is hidden from the other party). Examples of real-life private functions include airport no-fly check function, credit evaluation function, background- and medical history checking

\footnotetext{
^ The work was done while the author was visiting Bell Laboratories.
} 
function, etc. Full or even partial revelation of these functions opens vulnerabilities in the corresponding process, exploitable by dishonest participants (e.g. credit applicants), and should be prevented. It is known that the problem of PF-SFE can be reduced to the "regular" SFE 24/23]. This is done by evaluating a Universal Circuit (UC) 25 15] instead of a circuit defining the evaluated function. UC can be thought of as a "program execution circuit", capable of simulating any circuit $C$ of certain size, given the description of $C$ as input. Therefore, disclosing the UC does not reveal anything about $C$, except its size. At the same time, the SFE computes output correctly and $C$ remains private, since the player holding $C$ simply treats description of $C$ as additional (private) input to SFE. This reduction is the most common (and often the most efficient) way of securely evaluating private functions 242315.

\section{$1.1 \quad$ Related Work}

General SFE has been a subject of immense amount of research, started by Yao 27/28, which resulted in significant advances in the field 9 2117]. Fairplay [19] is a full practical implementation of general SFE based on garbled circuits.

Information-theoretic setting of SFE has also received a large amount of attention, e.g. 1311. However, due to the restrictions of the model, the resulting protocols are less efficient than those in the generous RO model. We apply some of the ideas of this setting, such as the efficient XOR gate construction (e.g. Construction 4 of [14]), in the RO setting, to obtain more efficient protocols.

\subsection{Our Contributions}

We present a new garbled circuit construction for two-party secure function evaluation (SFE) in the semi-honest model. In our one-round protocol, XOR gates are evaluated "for free" (that is, without the use of the associated garbled tables and the corresponding hashing or symmetric key operations). Our construction is as efficient as the best garbled circuit implementations (e.g. Fairplay 19]) in handling other gates.

We next show that free XOR gates bring significant benefit to many SFE settings. We show how to build permutation networks [26] and UC [25]15] almost exclusively of XOR gates; this results in a factor of up to 4 improvement (in both computation and communication) of their SFE. As discussed above, SFE of UC is the most efficient way of evaluating private functions; thus our work improves performance of PF-SFE almost fourfold. We note that other useful functions can benefit from free XOR gates. We show how to obtain a factor of up to 2 improvement of SFE of integer addition and equality testing.

We rely on the RO assumption; we discuss its (conservative) use in Sect. 3.1

\section{Setting and Preliminaries}

We consider acyclic boolean circuits with $k$ gates and arbitrary fan-out. That is, the (single) output of each gate can be used as input to an arbitrary number of 
gates. We assume that the gates $G_{1}, \ldots, G_{k}$ of the circuit are ordered topologically. This order (which is not necessarily unique) ensures that the $i$-th gate $G_{i}$ has no inputs that are outputs of a successive gate $G_{j}$, where $j>i$. A topological order can always be obtained on acyclic circuits, with $O(k)$ computation.

We concentrate on the semi-honest model, where players follow the protocol, but try to learn information from the execution transcripts.

We use the following standard notation: $\epsilon_{R}$ denotes uniform random sampling, || denotes concatenation of bit strings. $\langle a, b\rangle$ is a vector with two components $a$ and $b$, and its bit string representation is $a \| b . W_{c}=g\left(W_{a}, W_{b}\right)$ denotes a 2-input gate $G$ that computes function $g:\{0,1\}^{2} \rightarrow\{0,1\}$ with input wires $W_{a}$ and $W_{b}$ and output wire $W_{c}$.

Let $N$ be the security parameter. Let $S$ be an infinite set and let $X=$ $\left\{X_{s}\right\}_{s \in S}$ and $Y=\left\{Y_{s}\right\}_{s \in S}$ be distribution ensembles. We say that $\mathrm{X}$ and $\mathrm{Y}$ are computationally indistinguishable, denoted $X \stackrel{c}{\equiv} Y$, if for every non-uniform polynomial-time distinguisher $D$ and all sufficiently large $s \in S, \mid \operatorname{Pr}\left[D\left(X_{s}\right)=\right.$ 1] $-\operatorname{Pr}\left[D\left(Y_{s}\right)=1\right] \mid<1 / p(|s|)$ for every polynomial $p$.

Random Oracle. RO model is a useful abstraction, introduced and justified by [3]. RO is simply a randomly chosen function $\{0,1\}^{*} \mapsto\{0,1\}^{N}$ - a large object which cannot be fully stored or traversed by polytime players. RO model gives oracle access to such function to all players. In practice, ROs are modeled by hash functions, such as SHA. Although it was shown [5] that a protocol secure in the RO model may not be secure once RO is instantiated, "natural" RO protocols maintain their security in practice, and are widely used.

Oblivious Transfer (OT). The 1-out-of-2 OT is a two-party protocol. The sender $P_{1}$ has two secrets $m_{0}, m_{1}$, and the receiver $P_{2}$ has an selection bit $i \in$ $\{0,1\}$. At the end of the protocol, $P_{2}$ learns $m_{i}$, but nothing about $m_{1-i}$, and $P_{1}$ learns nothing about $i$. One-round OT is a widely studied primitive in the standard model [21], with improved implementations in the RO model [20]3.

Yao's Garbled Circuit (GC). The GC approach, excellently presented in [17, is the most efficient method of SFE of boolean circuits. Here we summarize its idea. Player $P_{1}$ first garbles circuit $C$ : for each wire $W_{i}$, he randomly chooses two secrets, $w_{i}^{0}$ and $w_{i}^{1}$, where $w_{i}^{j}$ is a garbled value, or garbling, of the $W_{i}$ 's value $j$. (Note: $w_{i}^{j}$ does not reveal $j$.) Further, for each gate $G_{i}, P_{1}$ creates and sends to $P_{2}$ a garbled table $T_{i}$, with the following property: given a set of garblings of $G_{i}$ 's inputs, $T_{i}$ allows to recover the garbling of the corresponding $G_{i}$ 's output, and nothing else. Then garblings of players' inputs are (obliviously) transferred to $P_{2}$. Now, $P_{2}$ can obtain the garbled output simply by evaluating the garbled circuit gate by gate, using the tables $T_{i}$. We call $W_{i}$ 's garbling $w_{i}^{j}$ active if $W_{i}$ assumes the value $j$ when $C$ is evaluated on the given input. Observe that for each wire, $P_{2}$ can obtain only its active garbling. The output wires of the circuit are not garbled (or their garblings are published), thus $P_{2}$ learns (only) the output of the circuit, and no internal wire values. $P_{1}$ learns the output from (semi-honest) $P_{2}$. (This step is trivial in the semi-honest model, and is usually not considered 
in the analysis.) Correctness of GC follows from method of construction of tables $T_{i}$. Neither party learns any additional information from the protocol execution.

\section{Our Protocol}

Overview. In our construction, we combine GC with the simple informationtheoretic SFE implementation of XOR-gates (e.g., Construction 4 of [14]). In all GC implementations, XOR gates cost as much as AND or OR gates (i.e. in computation and communication required for creation, transfer and evaluation of the garbled tables). The XOR gates of Kolesnikov [14 are free of these costs. However, his construction imposes a restrictive global relationship on the wire secrets, which prevents its use in previous GC schemes. In this work, we show how to overcome this restriction.

First, we show an SFE implementation of the XOR gate $G$, derived from one of 14. Let $G$ have two input wires $W_{a}, W_{b}$ and output wire $W_{c}$. Garble the wire values as follows. Randomly choose $w_{a}^{0}, w_{b}^{0}, R \in_{R}\{0,1\}^{N}$. Set $w_{c}^{0}=w_{a}^{0} \oplus w_{b}^{0}$, and $\forall i \in\{a, b, c\}: w_{i}^{1}=w_{i}^{0} \oplus R$. It is easy to see that the garbled gate output is simply obtained by XORing garbled gate inputs:

$w_{c}^{0}=w_{a}^{0} \oplus w_{b}^{0}=\left(w_{a}^{0} \oplus R\right) \oplus\left(w_{b}^{0} \oplus R\right)=w_{a}^{1} \oplus w_{b}^{1}$

$w_{c}^{1}=w_{c}^{0} \oplus R=w_{a}^{0} \oplus\left(w_{b}^{0} \oplus R\right)=w_{a}^{0} \oplus w_{b}^{1}=\left(w_{a}^{0} \oplus R\right) \oplus w_{b}^{0}=w_{a}^{1} \oplus w_{b}^{0}$. Further, garblings $w_{i}^{j}$ do not reveal the wire values they correspond to.

We can now pinpoint the restriction that the above XOR construction imposes on the garbled values - the garblings of the two values of each wire in the circuit must differ by the same value, i.e. $\forall i: w_{i}^{1}=w_{i}^{0} \oplus R$, for some global $R$. In contrast, in previous GC constructions, all garblings $w_{i}^{j}$ were chosen independently at random, and proofs of security relied on that property.

Our main observation is that it is not necessary to select all garblings independently. In our construction (Sect. 3.1), we choose a random $R$ once, and garble wire values, so that $\forall i: w_{i}^{1}=w_{i}^{0} \oplus R$.

\subsection{Our Garbled Circuit Construction}

Let $C$ be a circuit. We first note that NOT gates can be implemented "for free" by simply eliminating them and inverting the correspondence of the wires' values and garblings. We thus do not further consider NOT gates.

We implement XOR gates as discussed above in Sect. 3. Further, we replace each XOR-gate with $n>2$ inputs with $n-1$ two-input XOR-gates.

We implement all other gates using standard garbled tables [19. Namely, each gate with $n$ inputs is assigned a table with $2^{n}$ randomly permuted entries. Each entry is an encrypted garbling of the output wire, and garblings of the input wires serve as keys to decrypt the "right" output value. For simplicity, we present our construction and proof for the case $n=2$. The generalization to $n$-input gates $(n \geq 1)$ is straightforward.

In Alg. 1 below, each garbling $w=\langle k, p\rangle$ consists of a key $k \in\{0,1\}^{N}$ and a permutation bit $p \in\{0,1\}$. The key is used for decryption of the table entries, 
and $p$ is used to select the entry for decryption. The two garblings $w_{i}^{0}, w_{i}^{1}$ of each wire $W_{i}$ are related as required by the XOR construction: for a chosen $R \in_{R}\{0,1\}^{N}, \forall i: w_{i}^{1}=\left\langle k_{i}^{1}, p_{i}^{1}\right\rangle=\left\langle k_{i}^{0} \oplus R, p_{i}^{0} \oplus 1\right\rangle$, where $w_{i}^{0}=\left\langle k_{i}^{0}, p_{i}^{0}\right\rangle$. $H:\{0,1\}^{*} \mapsto\{0,1\}^{N+1}$ is a RO.

We now formalize the above intuition and present the GC construction (Alg. 11) and evaluation (Alg. 2). In SFE, Alg. 1 is run by $P_{1}$ and Alg. 2 is run by $P_{2}$.

Algorithm 1. (Construction of a garbled circuit)

1. Randomly choose global key offset $R \in R\{0,1\}^{N}$

2. For each input wire $W_{i}$ of $C$

(a) Randomly choose its garbled value $w_{i}^{0}=\left\langle k_{i}^{0}, p_{i}^{0}\right\rangle \in_{R}\{0,1\}^{N+1}$

(b) Set the other garbled output value $w_{i}^{1}=\left\langle k_{i}^{1}, p_{i}^{1}\right\rangle=\left\langle k_{i}^{0} \oplus R, p_{i}^{0} \oplus 1\right\rangle$

3. For each gate $G_{i}$ of $C$ in topological order

(a) label $G(i)$ with its index: label $\left(G_{i}\right)=i$

(b) If $G_{i}$ is an XOR-gate $W_{c}=X O R\left(W_{a}, W_{b}\right)$ with garbled input values $w_{a}^{0}=\left\langle k_{a}^{0}, p_{a}^{0}\right\rangle, w_{b}^{0}=\left\langle k_{b}^{0}, p_{b}^{0}\right\rangle, w_{a}^{1}=\left\langle k_{a}^{1}, p_{a}^{1}\right\rangle, w_{b}^{1}=\left\langle k_{b}^{1}, p_{b}^{1}\right\rangle:$

$i$. Set garbled output value $w_{c}^{0}=\left\langle k_{a}^{0} \oplus k_{b}^{0}, p_{a} \oplus p_{b}\right\rangle$

ii. Set garbled output value $w_{c}^{1}=\left\langle k_{a}^{0} \oplus k_{b}^{0} \oplus R, p_{a} \oplus p_{b} \oplus 1\right\rangle$

(c) If $G_{i}$ is a 2-input gate $W_{c}=g_{i}\left(W_{a}, W_{b}\right)$ with garbled input values $w_{a}^{0}=\left\langle k_{a}^{0}, p_{a}^{0}\right\rangle, w_{b}^{0}=\left\langle k_{b}^{0}, p_{b}^{0}\right\rangle, w_{a}^{1}=\left\langle k_{a}^{1}, p_{a}^{1}\right\rangle, w_{b}^{1}=\left\langle k_{b}^{1}, p_{b}^{1}\right\rangle:$

$i$. Randomly choose garbled output value $w_{c}^{0}=\left\langle k_{c}^{0}, p_{c}^{0}\right\rangle \in_{R}\{0,1\}^{N+1}$

ii. Set garbled output value $w_{c}^{1}=\left\langle k_{c}^{1}, p_{c}^{1}\right\rangle=\left\langle k_{c}^{0} \oplus R, p_{c}^{0} \oplus 1\right\rangle$

iii. Create $G_{i}$ 's garbled table. For each of $2^{2}$ possible combinations of $G_{i}$ 's input values $v_{a}, v_{b} \in\{0,1\}$, set

$$
e_{v_{a}, v_{b}}=H\left(k_{a}^{v_{a}}\left\|k_{b}^{v_{b}}\right\| i\right) \oplus w_{c}^{g_{i}\left(v_{a}, v_{b}\right)}
$$

Sort entries e in the table by the input pointers, i.e. place entry $e_{v_{a}, v_{b}}$ in position $\left\langle p_{a}^{v_{a}}, p_{b}^{v_{b}}\right\rangle$

4. For each circuit-output wire $W_{i}$ (the output of gate $G_{j}$ ) with garblings $w_{i}^{0}=\left\langle k_{i}^{0}, p_{i}^{0}\right\rangle, w_{i}^{1}=\left\langle k_{i}^{1}, p_{i}^{1}\right\rangle:$

(a) Create garbled output table for both possible wire values $v \in\{0,1\}$. Set

$$
e_{v}=H\left(k_{i}^{v} \| \text { "out" } \| j\right) \oplus v
$$

Sort entries $e$ in the table by the input pointers, i.e. place entry $e_{v}$ in position $p_{i}^{v}$. (There is no conflict, since $p_{i}^{1}=p_{i}^{0} \oplus 1$.)

Note, our encryption of table entries (Step 3(c)iii) is similar to that of Fairplay [19, Section 4.2]. Fairplay uses $e_{v_{a}, v_{b}}=H\left(k_{a}^{v_{a}}\|i\| p_{a}^{v_{a}} \| p_{b}^{v_{b}}\right) \oplus H\left(k_{b}^{v_{b}}\|i\| p_{a}^{v_{a}} \| p_{b}^{v_{b}}\right)$ $\oplus w_{c}^{g_{i}\left(v_{a}, v_{b}\right)}$. This is a non-essential difference; we could use Fairplay's encryption.

Intuition for security. (A formal proof is given in Sect. 3.2.) Alg. 1 uses the output of the RO $H$ as a one-time pad to encrypt the garbled output values in the garbled tables (Step 3(c)iii) and the garbled output tables (Step 4a). Note, any specific combination of $H$ 's inputs (keys and gate indices) is used for encryption of at most one table entry throughout our construction. (We assume 
that concatenation and string representation inside $H$ is done "right".) Further, since the evaluator of the garbled circuit only knows one garbled value per wire, he can decrypt exactly one entry of $G_{i}$ 's garbled table. All other entries are encrypted with at least one key that cannot be guessed by a polytime evaluator. Therefore, one of the two of garbled values of every wire looks random to him.

We now give the corresponding GC evaluation algorithm, run by $P_{2}$. Recall, $P_{2}$ obtains all garbled tables and the garblings of $P_{1}$ 's input values from $P_{1}$. Garblings of input values held by $P_{2}$ are sent via OT.

Algorithm 2. (Evaluation of a garbled circuit):

1. For each input wire $W_{i}$ of $C$

(a) Receive corresponding garbled value $w_{i}=\left\langle k_{i}, p_{i}\right\rangle$

2. For each gate $G_{i}$ (in the topological order given by labels)

(a) If $G_{i}$ is an XOR-gate $W_{c}=X O R\left(W_{a}, W_{b}\right)$ with garbled input values $w_{a}=\left\langle k_{a}, p_{a}\right\rangle, w_{b}=\left\langle k_{b}, p_{b}\right\rangle$

i. Compute garbled output value $w_{c}=\left\langle k_{c}, p_{c}\right\rangle=\left\langle k_{a} \oplus k_{b}, p_{a} \oplus p_{b}\right\rangle$

(b) If $G_{i}$ is a 2-input gate $W_{c}=g_{i}\left(W_{a}, W_{b}\right)$ with garbled input values $w_{a}=$ $\left\langle k_{a}, p_{a}\right\rangle, w_{b}=\left\langle k_{b}, p_{b}\right\rangle$

$i$. Decrypt garbled output value from garbled table entry e in position $\left\langle p_{a}, p_{b}\right\rangle: w_{c}=\left\langle k_{c}, p_{c}\right\rangle=H\left(k_{a}\left\|k_{b}\right\| i\right) \oplus e$

3. For each $C$ 's output wire $W_{i}$ (output of gate $G_{j}$ ) with garbling $w_{i}=\left\langle k_{i}, p_{i}\right\rangle$

(a) Decrypt output value $f_{i}$ from garbled output table entry e in row $p_{i}$ : $f_{i}=H\left(k_{i} \|\right.$ "out" $\left.\| j\right) \oplus e$

The GC construction and evaluation algorithms can be directly used to obtain the GC-based SFE protocol, in a standard manner. For completeness, we include the description of this protocol.

Protocol 1. (Two-party SFE protocol):

- Inputs: $P_{1}$ has private input $x=\left\langle x_{1}, . ., x_{u_{1}}\right\rangle \in\{0,1\}^{u_{1}}$ and $P_{2}$ has private input $y=\left\langle y_{1}, . ., y_{u_{2}}\right\rangle \in\{0,1\}^{u_{2}}$.

- Auxiliary input: $A$ boolean acyclic circuit $C$ such that $\forall x \in\{0,1\}^{u_{1}}, y \in$ $\{0,1\}^{u_{2}}$, it holds that $C(x, y)=f(x, y)$, where $f:\{0,1\}^{u_{1}} \times\{0,1\}^{u_{2}} \rightarrow$ $\{0,1\}^{v}$. We require that $C$ is such that if a circuit-output wire leaves some gate $G$, then gate $G$ has no other wires leading from it into other gates (i.e., no circuit-output wire is also a gate-input wire). Likewise, a circuit-input wire that is also a circuit-output wire enters no gates. We also require that $C$ is modified to contain no NOT-gates and all $n$-input XOR-gates with $n>2$ replaced by 2-input XOR-gates as described in Section 3.1 .

- The protocol:

1. $P_{1}$ constructs the garbled circuit using Algorithm 1 and sends it (i.e. the garbled tables) to $P_{2}$.

2. Let $W_{1}, . ., W_{u_{1}}$ be the circuit input wires corresponding to $x$, and let $W_{u_{1}+1}, \ldots, W_{u_{1}+u_{2}}$ be the circuit input wires corresponding to $y$. Then,

(a) $P_{1}$ sends $P_{2}$ the garbled values $w_{1}^{x_{1}}, . ., w_{u_{1}}^{x_{u_{1}}}$. 
(b) For every $i \in\left\{1, \ldots, u_{2}\right\}, P_{1}$ and $P_{2}$ execute a 1-out-of-2 oblivious transfer protocol, where $P_{1}$ 's input is $\left(k_{u_{1}+i}^{0}, k_{u_{1}+i}^{1}\right)$, and $P_{2}$ 's input is $y_{i}$. All $u_{2}$ OT instances can be run in parallel.

3. $P_{2}$ now has the garbled tables and the garblings of circuit's input wires. $P_{2}$ evaluates the garbled circuit, as described in Alg. 2, and outputs $f(x, y)$.

It is easy to verify protocol's correctness; we do not discuss it further.

On Our use of RO. In previous GC work, RO's use improves efficiency in the malicious model, but is not inherent. Here, while we rely on RO, we do so conservatively. First, we use non-programmable RO [22, i.e. we don't allow simulator to fake RO's answers. Second, (a variant of) correlation-robust functions [10], a weaker notion than RO, is sufficient for our purposes. (Recall, if $h$ is correlationrobust and $R, t_{1}, . ., t_{n}$ are random, $\left(h\left(t_{1} \oplus R\right), . ., h\left(t_{n} \oplus R\right)\right)$ is pseudo-random, given $t_{1}, . ., t_{n}$.)

Further, concrete security of our construction is comparable to that of standard GC with RO as the encryption function. This makes even constant-factor efficiency improvements, such as those suggested in this work, meaningful. For the lack of space, we omit the detailed analysis. We only note that the main feature of our protocol, the use of the global $R$, has very slight impact on security (e.g., our adversary can decrypt all garbled tables, once he breaks any one of them and learns $R$ ). Further, our use of RO is not vulnerable to birthday attacks in the semi-honest model. Indeed, the circuit is small, and $P_{2}$ w.h.p. will not see RO collisions.

\subsection{Proof of Security}

Our protocol is secure against semi-honest adversaries, who are not allowed to deviate from the protocol. Analogously to [1918], (w.h.p.) malicious behavior of players can be prevented by using cut-and-choose method; we don't discuss malicious players further.

We prove security in the simulation paradigm. Intuitively, a protocol $\pi$ is secure if whatever is seen by its party, can be computed only from that party's input and output. The view of a party $P_{i}$, view ${ }_{P_{i}}^{\pi}(x, y)$, consists of the party's own input, randomness, and all messages that $P_{i}$ receives in the execution of $\pi$. Thus, a protocol is secure, if there exist simulators $S_{1}, S_{2}$, such that $\left\{S_{1}(x, f(x, y)\} \stackrel{c}{\equiv}\right.$ $\left\{\right.$ view $\left._{P_{1}}^{\pi}(x, y)\right\}$ and $\left\{S_{2}(y, f(x, y)\} \stackrel{c}{\equiv}\left\{\right.\right.$ view $\left._{P_{2}}^{\pi}(x, y)\right\}$.

Case $1-\mathbf{P}_{1}$ is corrupted. $P_{1}$ 's view in Protocol 1 consists only of the view in the OT protocols in Step 2b, The following $S_{1}(x, f(x, y))$ simulates the view of $P_{1}$. Let $S_{1}^{O T}$ be the simulator that is guaranteed to exist for $P_{1}$ in the secure 1-out-of-2 OT protocol. $S_{1}$ constructs a garbled circuit using Alg. 1, Then $S_{1}$ feeds the constructed garblings of the input wires corresponding to $y$ to $S_{1}^{O T}$, and obtains the simulated transcript of the OT, which he outputs. $S$ additionally outputs $x$ and the randomness used in construction of GC. It is not hard to see that the output of the simulator is indistinguishable from the view of $P_{1}$. 
Case $2-\mathbf{P}_{\mathbf{2}}$ is corrupted. We construct a simulator $S_{2}$ that given input $(y, f(x, y))$ simulates the view of $P_{2} . P_{2}$ receives a garbled circuit (including garbled inputs), which $S_{2}$ must simulate. However, $S_{2}$ doesn't know $P_{1}$ 's input $x$. Thus, $S_{2}$ can not honestly generate the garbled circuit, since it doesn't know which of the input garblings corresponding to $x$ to hand to $P_{2}$ in Step 2a of the protocol. Instead, $S_{2}$ generates a fake garbled circuit that always evaluates to $f(x, y)$, using a slightly modified Alg. 1. The only modification, in Step 4a. appropriately forges the output tables:

4. For each circuit-output wire $W_{i}$ (the output of gate $G_{j}$ ) with garblings $w_{i}^{0}=\left\langle k_{i}^{0}, p_{i}^{0}\right\rangle, w_{i}^{1}=\left\langle k_{i}^{1}, p_{i}^{1}\right\rangle$ :

(a) Create fake garbled output table for both possible wire values $v \in\{0,1\}$ of the same encrypted output value. Set

$$
e_{v}=H\left(k_{i}^{v} \| \text { "out" } \| j\right) \oplus \mathbf{f}_{\mathbf{i}}(\mathbf{x}, \mathbf{y})
$$

Sort entries $e$ in the table by the input pointers, i.e. place entry $e_{v}$ in position $p_{i}^{v}$.

Let $S_{2}^{O T}$ be an OT simulator for $P_{2} . S_{2}$ outputs $y$, and the fake garbled circuit (i.e. its tables). Further, for each input wire $W_{i}$ held by $P_{2}, S_{2}$ runs and outputs $S_{2}^{O T}\left(y_{i}, w_{i}^{y_{i}}\right)$. Finally, $S_{2}$ simulates the received garblings of the input wires $W_{j}$ held by $P_{1}$ simply by outputting $w_{j}^{0}$ (fake garblings corresponding to $x=0 . .0$ ).

Theorem 1. The output of $S_{2}$ is indistinguishable from the real view of $P_{2}$.

Proof. (sketch) First, observe that $S_{2}$ feeds $S_{2}^{O T}$ proper inputs (i.e. $y$ and the corresponding honestly generated garblings). Thus, simulation of Step $2 \mathrm{~b}$ of the protocol is indistinguishable from the real execution. The crux of the proof is in showing the indistinguishability of the fake and real circuits (which include the tables and the input garblings that $P_{2}$ sees). This is addressed next.

First, observe, pointers $p_{i}^{j}$ are independent of the parties' inputs, and thus are easily simulated by $S_{2}$. For ease of presentation, we omit the details of pointer simulation from the proof.

We now show that no polytime procedure $D$ can distinguish simulated and real garbled circuit transcripts with non-negligible probability. We proceed inductively, gate by gate in topological order, in proving this for each partial transcript $\tau_{i}$, where $\tau_{0}$ includes all active secrets on the input wires, and each $\tau_{i}$ additionally includes the garbled tables of first $i$ gates.

Induction base. It is easy to see that the partial transcript $\tau_{0}-$ active secrets on the input wires - is distributed identically in real and simulated cases. Indeed, these secrets are uniformly random in the domain. Moreover, clearly, no distinguisher $D_{0}$ can output with non-negligible probability the global key offset $\hat{R}$ used in the construction of the (either simulated or real) transcript.

For the induction step, suppose no polytime $D_{i-1}$ can with non-negligible advantage distinguish the $\tau_{i-1}$ transcripts (i.e. those including the active secrets on the inputs and the first $i-1$ garbled tables). Moreover, assume that no 
polytime $D_{i-1}$ can output the global key offset $\hat{R}$ with non-negligible probability when given $\tau_{i-1}$. We show that these properties hold also when additionally given the $i$-th garbled table.

Recall, the $i$-th garbled table contains (a permutation of) entries:

$$
\begin{aligned}
& H\left(k_{a}\left\|k_{b}\right\| i\right) \oplus v_{00} \\
& H\left(k_{a}\left\|k_{b} \oplus \hat{R}\right\| i\right) \oplus v_{01} \\
& H\left(k_{a} \oplus \hat{R}\left\|k_{b}\right\| i\right) \oplus v_{10} \\
& H\left(k_{a} \oplus \hat{R}\left\|k_{b} \oplus \hat{R}\right\| i\right) \oplus v_{11}
\end{aligned}
$$

where $v_{00}, . ., v_{11} \in\left\{k_{c}, k_{c} \oplus \hat{R}\right\}$ are the output secrets that correspond to the four possible gate input combinations. (Garbled output tables have one input and consist of two entries. The corresponding claims hold for these cases as well, via a natural modification of the following argument addressing two-input gates.)

Without loss of generality, suppose the active gate input secrets are $k_{a}$ and $k_{b}$. By the induction assumption, no polytime $D_{i-1}$ can compute both $k_{a}$ and $k_{a} \oplus \hat{R}$, or both $k_{b}$ and $k_{b} \oplus \hat{R}$ (otherwise $D_{i-1}$ can output $\hat{R}$ ). Thus, $D_{i-1}$ can call functions $H\left(k_{a}\left\|k_{b} \oplus \hat{R}\right\| i\right), H\left(k_{a} \oplus \hat{R}\left\|k_{b}\right\| i\right)$, or $H\left(k_{a} \oplus \hat{R}\left\|k_{b} \oplus \hat{R}\right\| i\right)$ only with negligible probability. Further, because of the inclusion of the gate index $i$, these function calls have not been made in the construction of (real or simulated) $\tau_{i}$. Therefore, due to RO properties, except with negligible probability, all the inactive entries in the $i$-th table are distributed identically to random strings, from the point of view of $D_{i-1}$, and thus do not provide help to $D_{i-1}$ in computing $\hat{R}$. Therefore, polytime $D_{i}$ cannot output $\hat{R}$ or call any of $H\left(k_{a}\left\|k_{b} \oplus \hat{R}\right\| i\right), H\left(k_{a} \oplus \hat{R}\left\|k_{b}\right\| i\right)$, or $H\left(k_{a} \oplus \hat{R}\left\|k_{b} \oplus \hat{R}\right\| i\right)$, except with negligible probability. Therefore, no polytime $D_{i}$ can distinguish the real and simulated transcripts $\tau_{i}$ with non-negligible probability.

This completes the induction and the proof of the theorem.

\section{Application of Our SFE Constructions}

We now present several motivating examples - practical functions whose SFE benefits from improvements of our construction. Universal circuit (UC) constructions 25 15] do not explicitly use many XOR gates. We show how to modify these circuits to mainly consists of XOR gates, achieving fourfold reduction of garbled circuit size. This construction may be of independent interest. Further, we show how to reduce in half the size of garbled circuits of commonly used blocks, such as integer addition and equality test.

Universal Circuits 25,15] and Permutation Networks [26]. The size of a UC mainly comes from programmable switching networks (such as permutation network 26]) connecting the simulated gates. In turn, these networks are constructed from two types of switching blocks shown in Fig. 1, as discussed in 26 25 15]. The $Y$-block can be programmed to output one of its two inputs. The $X$-block can be programmed to either pass or cross over its two inputs to the two outputs. A natural SFE implementation of the $Y$-block uses a 2-input garbled gate with a garbled table with $2^{2}=4$ encrypted table entries. Similarly, $X$-block 
A) B)

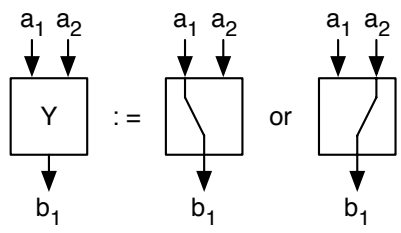

(a) $Y$ switching block
A) B)

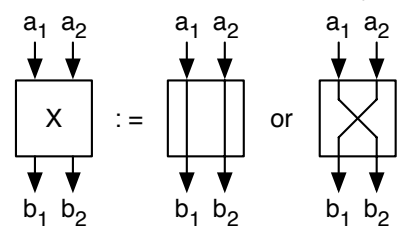

(b) $X$ switching block

Fig. 1. Switching blocks

is implemented by two 2-input garbled gates (one for each of its two outputs), resulting in a garbled table of $2 \cdot 2^{2}=8$ entries.

We show how to take advantage of free XOR gates and implement both $X$ and $Y$-gates with only two garbled table entries each. Since permutation network 26. consists only of $X$-gates, this results in $75 \%$ size reduction of its SFE. UC consists almost exclusively of $X$-gates. Valiant's UC [25] for a circuit of $k$ gates has size $\sim 19 k \log k$. The $\sim 19 k \log k-k$ overhead gates are $X$-gates that come from switching networks. A recent UC construction [15] similarly consists almost exclusively of $X$-gates, and of very few $Y$-gates and simulated gates. Thus, UC enjoys almost $75 \%$ garbled table size reduction.

Let $f:\{0,1\} \mapsto\{0,1\}$ be a function (implemented with two garbled table entries). We implement $X$ - and $Y$-blocks as follows (see Fig. 2). $Y\left(a_{1}, a_{2}\right)=b_{1}=$ $f\left(a_{1} \oplus a_{2}\right) \oplus a_{1} ; X\left(a_{1}, a_{2}\right)=\left(b_{1}, b_{2}\right)$, where $b_{1}=f\left(a_{1} \oplus a_{2}\right) \oplus a_{1}, b_{2}=f\left(a_{1} \oplus a_{2}\right) \oplus$ $a_{2}$. It is easy to see that setting $f=f_{0}$ to the zero function results in $Y$ choosing left input, and $X$ passing the inputs. Further, setting $f=f_{i d}$ to the identity function results in $Y$ choosing the right input, and in $X$ crossing its inputs:

$$
\begin{aligned}
& f=f_{0}: \quad b_{1}=0 \oplus a_{1}=a_{1} ; \quad b_{2}=0 \oplus a_{2}=a_{2} . \\
& f=f_{i d}: \quad b_{1}=\left(a_{1} \oplus a_{2}\right) \oplus a_{1}=a_{2} ; \quad b_{2}=\left(a_{1} \oplus a_{2}\right) \oplus a_{2}=a_{1} \text {. }
\end{aligned}
$$

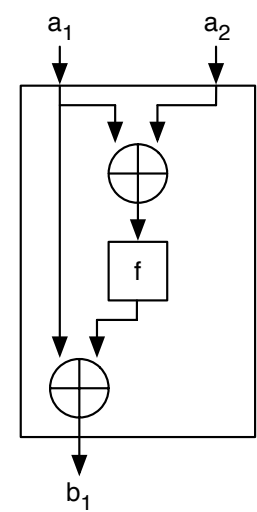

(a) $Y$ switching block

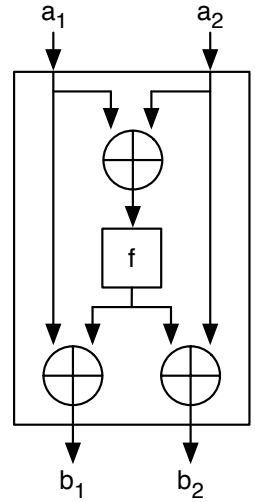

(b) $X$ switching block

Fig. 2. Efficient implementation of switching blocks 
This construction can be extended to implement programmable switching blocks $X$ and $Y$, which take an additional programming input bit $p$. This bit determines behavior of $X$ - (pass or cross) and $Y$-blocks (left or right input). The natural construction for the $Y$ - (resp. $X$-) switching block uses one (resp. two) 3-input gate(s) with $2^{3}=8$ (resp. 16) encrypted table entries. In our XOR-based construction, function $f$ is then replaced by a two-input AND-gate (with $p$ being the second input) with $2^{2}=4$ encrypted table entries. Clearly, $p=0$ sets $f=f_{0}$, and $p=1$ sets $f=f_{i d}$, allowing to program $X$ - and $Y$-blocks. As above, the size of $Y$ - and $X$-blocks is reduced by $50 \%$ and $75 \%$ respectively.

Integer Adder and Multiplier. An adder for $n$-bit integers $a, b$ is composed from a chain of $n$ full adder (FA) blocks as shown in Fig. 3(b) (The last FA block can be replaced by a smaller half-adder block.) A FA block (see Fig. 3(a) has as inputs a carry-in $c_{i}$ from the previous FA block and the two input bits $a_{i}$ and $b_{i}$. It outputs two bits: carry-out $c_{i+1}=\left(a_{i} \wedge b_{i}\right) \vee\left(a_{i} \wedge c_{i}\right) \vee\left(b_{i} \wedge c_{i}\right)$ and sum $s_{i}=a_{i} \oplus b_{i} \oplus c_{i}$. The straightforward implementation of a FA uses two 3 -input gates with $2 \cdot 2^{3}=16$ encrypted table entries. We can compute $s_{i}$ "for free" using free XOR-gates, and use only one 3 -input gate with $2^{3}=8$ encrypted table entries to compute $c_{i+1}$. The size of a FA block, and hence that of an $n$-bit adder is reduced by $50 \%$.

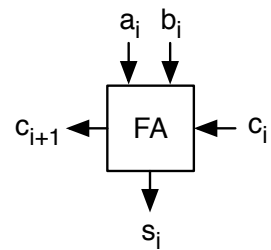

(a) Full Adder (FA)

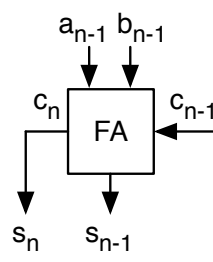

(b) n-bit Adder built from n FA blocks

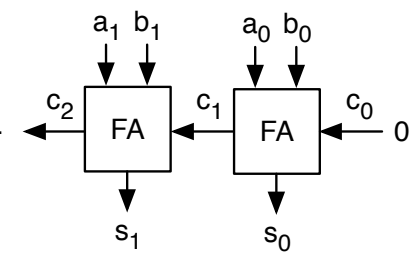

Fig. 3. Adder for two n-bit integers a and b

As circuits for integer multiplication consist of bit-multipliers (2-input ANDgates) and adders, the improved implementation of adders can directly be used to correspondingly improve integer-multiplication circuits.

Integer Equality Test. A similar construction is used to test equality of two $n$ bit integers $a$ and $b$. Now, we do not compute $s_{i}$, and use carry bits as inequality flags. The carry-out bit is defined as $c_{i+1}=\left(a_{i} \neq b_{i}\right) \vee c_{i}=\left(a_{i} \oplus b_{i}\right) \vee c_{i}$. A simple implementation uses two 2-input gates or one 3 -input gate (each costs 8 encrypted table entries). Free XOR gate reduces the cost to that of one 2input OR gate (4 encrypted table entries). The size of equality test block is thus reduced by $50 \%$.

Acknowledgments. We thank Yuval Ishai and anonymous referees for helpful comments. The second author thanks CACE project for funding his ICALP trip. 


\section{References}

1. Aiello, W., Ishai, Y., Reingold, O.: Priced oblivious transfer: How to sell digital goods. In: Pfitzmann, B. (ed.) EUROCRYPT 2001. LNCS, vol. 2045, pp. 119-135. Springer, Heidelberg (2001)

2. Bellare, M., Micali, S.: Non-interactive oblivious transfer and applications. In: Brassard, G. (ed.) CRYPTO 1989. LNCS, vol. 435, pp. 547-557. Springer, Heidelberg (1990)

3. Bellare, M., Rogaway, P.: Random oracles are practical: A paradigm for designing efficient protocols. In: ACM CCS, pp. 62-73 (1993)

4. Blake, I.F., Kolesnikov, V.: Conditional encrypted mapping and comparing encrypted numbers. In: Di Crescenzo, G., Rubin, A. (eds.) FC 2006. LNCS, vol. 4107, pp. 206-220. Springer, Heidelberg (2006)

5. Canetti, R., Goldreich, O., Halevi, S.: The random oracle methodology, revisited. In: Proc. 30th ACM Symp. on Theory of Computing, pp. 209-218 (1998)

6. Crescenzo, G.D.: Private selective payment protocols. In: Frankel, Y. (ed.) FC 2000. LNCS, vol. 1962. Springer, Heidelberg (2001)

7. Even, S., Goldreich, O., Lempel, A.: A randomized protocol for signing contracts. Commun. ACM 28(6), 637-647 (1985)

8. Fischlin, M.: A cost-effective pay-per-multiplication comparison method for millionaires. In: Naccache, D. (ed.) CT-RSA 2001. LNCS, vol. 2020, pp. 457-471. Springer, Heidelberg (2001)

9. Goldreich, O., Vainish, R.: How to solve any protocol problem - an efficiency improvement. In: Pomerance, C. (ed.) CRYPTO 1987. LNCS, vol. 293, pp. 73-86. Springer, Heidelberg (1988)

10. Ishai, Y., Kilian, J., Nissim, K., Petrank, E.: Extending oblivious transfers efficiently. In: Boneh, D. (ed.) CRYPTO 2003. LNCS, vol. 2729. Springer, Heidelberg (2003)

11. Ishai, Y., Kushilevitz, E.: Perfect constant-round secure computation via perfect randomizing polynomials. In: Widmayer, P., Triguero, F., Morales, R., Hennessy, M., Eidenbenz, S., Conejo, R. (eds.) ICALP 2002. LNCS, vol. 2380, pp. 244-256. Springer, Heidelberg (2002)

12. Kantarcioglu, M., Clifton, C.: Privacy-preserving distributed mining of association rules on horizontally partitioned data. In: ACM SIGMOD Workshop on Research Issues on Data Mining and Knowledge Discovery (DMKD 2002) (2002)

13. Kilian, J.: Founding cryptography on oblivious transfer. In: Proc. 20th ACM Symp. on Theory of Computing, Chicago, pp. 20-31. ACM, New York (1988)

14. Kolesnikov, V.: Gate evaluation secret sharing and secure one-round two-party computation. In: Roy, B. (ed.) ASIACRYPT 2005. LNCS, vol. 3788, pp. 136-155. Springer, Heidelberg (2005)

15. Kolesnikov, V., Schneider, T.: A practical universal circuit construction and secure evaluation of private functions. In: Financial Cryptography and Data Security, FC 2008. LNCS. Springer, Heidelberg (2008)

16. Lindell, Y., Pinkas, B.: Privacy preserving data mining. In: Bellare, M. (ed.) CRYPTO 2000. LNCS, vol. 1880. Springer, Heidelberg (2000)

17. Lindell, Y., Pinkas, B.: A proof of Yao's protocol for secure two-party computation. Cryptology ePrint Archive, Report 2004/175 (2004)

18. Lindell, Y., Pinkas, B.: An efficient protocol for secure two-party computation in the presence of malicious adversaries. In: Naor, M. (ed.) EUROCRYPT 2007. LNCS, vol. 4515, pp. 52-78. Springer, Heidelberg (2007) 
19. Malkhi, D., Nisan, N., Pinkas, B., Sella, Y.: Fairplay — a secure two-party computation system. In: USENIX (2004)

20. Naor, M., Pinkas, B.: Efficient oblivious transfer protocols. In: SODA 2001: Proceedings of the twelfth annual ACM-SIAM symposium on Discrete algorithms, Philadelphia, PA, USA. Society for Industrial and Applied Mathematics (2001)

21. Naor, M., Pinkas, B., Sumner, R.: Privacy preserving auctions and mechanism design. In: 1st ACM Conf. on Electronic Commerce (1999)

22. Nielsen, J.B.: Separating random oracle proofs from complexity theoretic proofs: The non-committing encryption case. In: Yung, M. (ed.) CRYPTO 2002. LNCS, vol. 2442, pp. 111-126. Springer, Heidelberg (2002)

23. Pinkas, B.: Cryptographic techniques for privacy-preserving data mining. SIGKDD Explor. Newsl. 4(2), 12-19 (2002)

24. Sander, T., Young, A., Yung, M.: Non-interactive cryptocomputing for $N C^{1}$. In: Proc. 40th FOCS, New York, pp. 554-566. IEEE, Los Alamitos (1999)

25. Valiant, L.G.: Universal circuits (preliminary report). In: Proc. 8th ACM Symp. on Theory of Computing, pp. 196-203. ACM Press, New York (1976)

26. Waksman, A.: A permutation network. J. ACM 15(1), 159-163 (1968)

27. Yao, A.C.: Protocols for secure computations. In: Proc. 23rd IEEE Symp. on Foundations of Comp. Science, Chicago, pp. 160-164. IEEE, Los Alamitos (1982)

28. Yao, A.C.: How to generate and exchange secrets. In: Proc. 27th IEEE Symp. on Foundations of Comp. Science, Toronto, pp. 162-167. IEEE, Los Alamitos (1986) 